\title{
Susceptibility of Maize to Stalk Rot Caused by Fusarium graminearum Deoxynivalenol and Zearalenone Mutants
}

\author{
L. M. Quesada-Ocampo, J. Al-Haddad, A. C. Scruggs, C. R. Buell, and F. Trail
}

First and third authors; Department of Plant Pathology, North Carolina State University, Raleigh 27695; and second, fourth, and fifth authors: Department of Plant Biology, Michigan State University, East Lansing 48824.

Accepted for publication 28 March 2016.

\begin{abstract}
Quesada-Ocampo, L. M., Al-Haddad, J., Scruggs, A. C., Buell, C. R., and Trail, F. 2016. Susceptibility of maize to stalk rot caused by Fusarium graminearum deoxynivalenol and zearalenone mutants. Phytopathology 106:920-927.

Fusarium graminearum is a destructive pathogen of cereals that can cause stalk rot in maize. Stalk rot results in yield losses due to impaired grain filling, premature senescence, and lodging, which limits production and harvesting of ears. In addition, mycotoxins can make infected tissues unfit for silage. Our objectives were to evaluate the natural variation in stalk rot resistance among maize inbreds, to establish whether deoxynivalenol (DON)- and zearalenone (ZEA)-deficient strains are pathogenic on a panel of diverse inbreds, and to quantify the accumulation of DON in infected stalk tissue. Wild-type $F$. graminearum and mycotoxin mutants

(DON and ZEA) were used to separately inoculate stalks of 9-week-old plants of 20 inbreds in the greenhouse. Plants were evaluated for lesion area at the inoculation point at $0,2,14$, and 28 days postinoculation and tissues around lesions were sampled to determine the DON content. Regardless of their ability to produce DON or ZEA, all tested $F$. graminearum strains caused stalk rot; however, significant differences in disease levels were detected. Among the tested inbreds, Mp717 was resistant to all three $F$. graminearum strains while Mp317 and HP301 were only partially resistant. Accumulation of DON was significantly lower in infected stalks of the resistant and partially resistant inbreds than the susceptible inbreds. Analysis of the 20 inbreds using data from 17 simple-sequence repeats revealed population structure among the individuals; however, there was no association between genetic clustering and stalk rot resistance. These findings are an additional step toward breeding maize inbreds suitable for planting in fields infested with $F$. graminearum.
\end{abstract}

Fusarium graminearum Schwabe (teleomorph Gibberella zeae (Schwein.) Petch) is a destructive fungal pathogen of cereal crops that can cause ear and stalk rot in maize (Kazan et al. 2012). Significant losses occur in maize when stalks die prematurely and plants produce lightweight ears with poorly filled kernels, or when stalk lodging occurs, which results in ear harvesting failures (Harris et al. 1999; Kazan et al. 2012; Sutton 1982). Stalk rot may cause further losses because severely infected tissue may contain significant levels of mycotoxins that are hazardous to humans and animals when ingested, making the stalks unacceptable for use as silage (Goswami and Kistler 2004; Mirocha and Christensen 1974).

In the field, $F$. graminearum is managed by reducing inoculum through crop rotation and controlling insect wounding that could facilitate pathogen entry and infection. Once a field is infested, few options are available to reduce disease incidence; harvesting early and drying the ears to prevent further fungal growth is advised (Goswami and Kistler 2004; Sutton 1982). The use of resistant inbreds is also recommended but commercial hybrids are not highly resistant and are mostly susceptible or intermediately resistant to $F$. graminearum (Munkvold 2003). The interaction of $F$. graminearum with maize has not been as intensively studied as the interaction with wheat, and there is little information about mycotoxin production, accumulation, and its possible correlation with stalk rot resistance (Atlin et al. 1983; Gendloff et al. 1986; Hart et al. 1982; Trail 2009). Previous research has identified

Corresponding author: L. M. Quesada-Ocampo;

E-mail address: lmquesad@ncsu.edu

*The $\boldsymbol{e}$-Xtra logo stands for "electronic extra" and indicates that two supplementary tables are published online.

http://dx.doi.org/10.1094/PHYTO-09-15-0199-R

(C) 2016 The American Phytopathological Society quantitative trait loci controlling resistance to $F$. graminearum stalk rot in the maize inbred 1145 (Ye et al. 2013). Identification of additional sources of resistance to $F$. graminearum stalk rot that display low levels of stalk rot would facilitate breeding of improved maize inbreds.

F. graminearum produces several mycotoxins, including deoxynivalenol (DON), nivalenol (NIV), zearalenone (ZEA), fusarin C, fusaric acid, and aurofusarin (Trail 2009). The development of an efficient transformation system for $F$. graminearum has facilitated gene functional analysis, and several knockout mutants for genes involved in toxin production are available (Baldwin et al. 2010; Dufresne et al. 2008; Gaffoor and Trail 2006; Gaffoor et al. 2005; Proctor et al. 1995; Seong et al. 2005). Experiments with $F$. graminearum mycotoxin mutants have shown that DON is a potent phytotoxin and virulence factor in wheat (Desjardins et al. 1996), and that it is differentially produced in diverse wheat tissues (Mudge et al. 2006). Comparative studies performed in barley and wheat established that genes expressed during $F$. graminearum infection are substantially different on diverse hosts, which highlights the importance of host-specific studies (Lysøe et al. 2011). Studies of maize ear rot caused by $F$. gramineraum mycotoxin mutants have found that DON is also a virulence factor in maize (Harris et al. 1999); however, no experiment has been performed to determine whether this is also the case for stalk rot, though a few studies have reported mycotoxin accumulation in maize stalks (Dorn et al. 2011; Eckard et al. 2011).

The objective of this study was to determine whether different maize inbreds show resistance to $F$. graminearum stalk rot and DON accumulation. Specifically, we sought to determine (i) if the evaluated inbreds could be used as a source of resistance to $F$. graminearum stalk rot, (ii) if DON and ZEA mutants have reduced virulence in maize when causing stalk rot, (iii) if inbreds resistant to $F$. graminearum stalk rot display a reduced accumulation of DON in infected tissue in comparison with more susceptible 
inbreds, and (iv) the association between inbred population structure and resistance to $F$. graminearum.

\section{MATERIALS AND METHODS}

Plant material. In total, 20 maize inbreds were evaluated for susceptibility to F. graminearum stalk rot (Supplementary Table $\mathrm{S} 1)$. Inbreds included 13 parents of the nested association mapping population (B73, B97, HP301, IL14H, Ky21, M162W, M37W, Mo18W, MS71, OH43, OH7B, P39, and Tx303) (McMullen et al. 2009), 4 inbreds that have shown resistance to $F$. graminearum ear rot in the field (CO441, GEMS-0002, Mp717, and NC444) (Mohammadi et al. 2011), 2 inbreds that are tolerant to ear rot in the field (GEMS0030 and Mp317), and 1 inbred that is highly susceptible to ear rot in the field (LH132) according to the Germplasm Enhancement of Maize (GEM) project (http://www.public.iastate.edu/ usda-gem/). Seed were treated with $2.7 \%$ sodium hypochlorite for $15 \mathrm{~min}$, rinsed with sterile water, and immediately sown on 72-square-cell plastic flats with cell depth of $5.7 \mathrm{~cm}$ and cell width of $4 \mathrm{~cm}$ (Hummert International, Earth City, MO), containing soilless medium (Baccto Professional Planting Mix; Michigan Peat Company, Houston, TX). Plants were grown in a research glass greenhouse for 3 weeks under 14-h day illumination, at a temperature setting of 26 and $22^{\circ} \mathrm{C}$ (day and night, respectively), and transferred into 7.5-liter square pots (Hummert International) containing the same soilless medium mixed with Osmocote 14-14-14 fertilizer (The Scotts Company, Marysville, $\mathrm{OH})$. Plants were allowed to recover from transplant stress for 5 weeks prior to being inoculated at the V8 stage and were irrigated as needed.

Fungal strains and inoculations. F. graminearum isolates included a wild-type strain (PH-1; NRRL31084; FGSC9075) and mutants for the mycotoxins DON $(\Delta$ tri5) and ZEA (double mutant $\Delta$ zea1 $\Delta$ zea2) derived from the wild-type PH-1 strain (Gaffoor et al. 2005). Isolate PH-1 was obtained from maize kernels in Michigan (Trail and Common 2000), is highly pathogenic on wheat, produces high levels of DON and ZEA on wheat (Gaffoor et al. 2005), and was the strain used in the $F$. graminearum genome sequencing project (Cuomo et al. 2007). Fresh, actively growing cultures of each strain were obtained by transferring agar plugs from long-term stock cultures onto potato dextrose agar (PDA) (DOT Scientific, Burton, MI). Cultures were grown on PDA at room temperature $\left(21 \pm 2^{\circ} \mathrm{C}\right)$ under fluorescent light for 4 days prior to inoculations. Agar plugs ( $5 \mathrm{~mm}$ in diameter) of actively growing $F$. graminearum containing mycelium and conidia were used for stalk inoculations. After the sheath tissue on the stalk was stripped from 12 plants per strain, a small wound ( $1 \mathrm{~mm}$ in diameter) was made in the middle of the stalk area between the soil line and the internode above it using a sterile syringe needle to simulate insect wounding. Then, the agar mycelial plug was placed face down over the wound and covered with parafilm to maintain a moist environment. As a control, 12 plants were inoculated as described with a sterile 5-mm-diameter agar plug from PDA. This experiment was conducted twice.

Disease assessment, tissue sampling, and statistic analysis. Disease assessment and tissue sampling were conducted at $0,2,14$, and 28 days postinoculation (dpi) for each treatment (control, PH1, $\Delta$ tri5, and $\Delta$ zea $1 \Delta$ zea2). Six plants were rated at the four time points and two of these plants, representative of the symptoms observed at a particular time point, were sampled for stalk tissue for DON quantification and for reisolation of $F$. graminearum strains in PDA agar to verify the inoculations. Stalk tissue was collected by cutting $2 \mathrm{~cm}$ below and $2 \mathrm{~cm}$ above the inoculation point after removal of the parafilm and plug, dividing the sample longitudinally in two. One half of the sample was refrigerated for subsequent pathogen isolation and the other half was flash frozen in liquid nitrogen and stored at $-80^{\circ} \mathrm{C}$ for DON quantification. Disease progression was recorded for each plant by measuring the horizontal and vertical lesion diameters to calculate the area of the oval lesion. The lesion area data were used to calculate the area under the disease progress curve (AUDPC) with the trapezoidal method, and data residuals fulfilled normality and variance assumptions for statistical analyses. Combined AUDPC data from the two replicated experiments were subjected to analysis of variance (ANOVA) using R (R Core Team 2013), and multiple comparisons among the means were conducted using Tukey's test when effects were found to be statistically significant $(P<0.05)$. Maize inbreds were categorized as resistant, moderately resistant, and susceptible to stalk rot based on results of multiple comparisons among the means using Tukey's test, where inbreds in the same category were not significantly different from each other.

DON quantification and statistic analysis. Targeted metabolomics was performed to measure levels of DON in maize inbreds representative of the disease responses observed (Supplementary Table S2). Inbreds B73, HP301, IL14H, Mp317, Mp717, and $\mathrm{NC} 444$ were measured for DON content when infected with each of the three isolates at $28 \mathrm{dpi}$. DON mean values at $28 \mathrm{dpi}$ were subjected to ANOVA and Pearson's correlation with AUDPC lesion data using R (R Core Team 2013). Multiple comparisons among the means were conducted using Tukey's test when effects were found to be statistically significant in ANOVA. Sampled tissue was lyophilized overnight and pulverized to powder using sterile stainless-steel beads and a paint shaker. Metabolites were extracted using 4:1 methanol/water with $1 \%$ acetic acid, in a ratio of 10:1 solvent/sample mass for normalization of samples. After adding the solvent, samples were mixed by vortexing and transferred to a $-20^{\circ} \mathrm{C}$ freezer overnight. Samples were centrifuged at $3,000 \times \mathrm{g}$ for $5 \mathrm{~min}$ at $4^{\circ} \mathrm{C}$, and supernatants (100 to $500 \mu \mathrm{l}$ in volume) were transferred to a new tube for storage and metabolite measurements. Measurements of DON concentration were performed in $50 \mu \mathrm{l}$ of the samples using a Waters Quattro micro API LC/MS/MS (Waters, Milford, MA) and a BetaBasic C-18 column (Thermo Scientific, Waltham, MA), with 9:1 formic acid 0.1\%/methanol as buffers, 3 -min runs, collision energy of $16 \mathrm{~V}$, and in positive mode. Optimal conditions for DON detection were determined using QuanOptimize (Waters). A calibration curve for DON was generated with QuanLynx (Waters) using a DON toxin standard (Sigma-Aldrich, St. Louis) diluted at $0.01,0.05,0.1,1,5$, and $10 \mu \mathrm{M}$. DON was identified in the sample by comparing $\mathrm{m} / \mathrm{z}$ values with the toxin standard, and toxin concentrations in the samples were quantified using QuanLynx (Waters) and the calibration curve. A toxin standard $(1 \mu \mathrm{M})$, buffer control, and blank were included in each row of 96-well plates containing samples for measurements for quality control.

Plant DNA extraction, microsatellite amplification, and genotyping. Seedlings of each inbred were harvested and stored at $-80^{\circ} \mathrm{C}$ before use. Plant tissues were ground in liquid nitrogen and the nuclear DNA was extracted using the DNeasy plant mini kit following the manufacturer's instructions (Qiagen, Valencia, CA). The DNA concentration for each sample was determined using a NanoDrop 100 spectrophotometer and the NanoDrop 2.4.7c software (Nano-Drop Technologies Inc., Wilmington, DE). A genomic DNA concentration of $15 \mathrm{ng} / \mu \mathrm{l}$ was used for amplification of 17 simplesequence repeats (SSR) (Table 1) selected from the MaizeGDB website and previous publications (Lawrence et al. 2004; Romay et al. 2012; Warburton et al. 2007). Forward primers were designed to include an M13 tail for fluorescent labeling of products and later fragment analyses (Schuelke 2000). Briefly, each polymerase chain reaction (PCR) was performed in $10 \mu \mathrm{l}$ of $2 \times$ GoTaq Hot Start Green Master Mix (Promega Corp., Madison, WI), $1 \mu \mathrm{l}$ of $10 \mu \mathrm{M}$ forward primer, $10 \mu \mathrm{M}$ reverse primer, $10 \mathrm{ng}$ of DNA, and sterile water. The PCR program was $3 \mathrm{~min}$ at $94^{\circ} \mathrm{C}$ for an initial denaturation; 35 cycles of $30 \mathrm{~s}$ at $94^{\circ} \mathrm{C}, 45 \mathrm{~s}$ at $53^{\circ} \mathrm{C}$, and $45 \mathrm{~s}$ at $72^{\circ} \mathrm{C}$; followed by a final extension at $72^{\circ} \mathrm{C}$ by $5 \mathrm{~min}$. PCR products amplified with the polymorphic SSR primers were subjected to a second round of PCR for fluorescent labeling (Schuelke 2000). Reactions were carried out in a 10- $\mu$ l volume as described above, with the exception of an M13 primer labeled with fluorescent dye in place of the site-specific forward primer (Schuelke 2000). Thermal cycler settings were the 
same as described above. Products from the second PCR were diluted 50 -fold and multiplexed, combining multiple PCR products of different SSR primers labeled with different fluorescent dyes (VIC and 6FAM) (Applied Biosystems, Foster City, CA). A genotyping reaction was performed where HiDi Formamide, LIZ600 size standard, and the diluted, multiplexed sample were combined. Samples were genotyped with a 3730xl DNA Analyzer (Applied Biosystems) and labeled fragments were analyzed in Peak Scanner (Applied Biosystems).

Genetic diversity and population structure analysis. Genetic diversity was estimated using the Mantel test and significance at each locus was determined with 1,000 permutations using the Exact test implemented in Powermarker v3.25 (Liu and Muse 2005). Genetic distance matrix values were calculated using Euclidean distance with the unweighted pair-group method with arithmetic mean (UPGMA) and visualized in MEGA6 (Tamura et al. 2013). Population structure of maize inbreds was assessed with the model-based Bayesian clustering algorithm implemented in Structure 2.3X. The values for burnin, chain replication, and $\lambda$ were set at 300,000,100,000 and 1, respectively, based on results obtained in preliminary analyses. The optimal number of populations $(K)$ was determined by comparing posterior distribution likelihoods among three independent runs from $K=1$ to $K=20$ using the established parameters. Data included 17 polymorphic SSR and were analyzed under the admixture model with correlated allele frequencies and without previous population information. Population structure figures were sorted by Q to establish associations between population structure and resistance to $F$. graminearum stalk rot. Wright's subpopulation fixation index and overall significance were determined using 1,000 bootstrap replicates $(2.5$ and $97.5 \%$ confidence intervals, $P=0.05$ ), as implemented in Powermarker.

\section{RESULTS}

The first disease symptoms occurred 2 dpi and progressed throughout the duration of the experiment at different rates, depending on the susceptibility of the maize inbred. Overall disease symptoms included necrotic lesions starting at the inoculation point and expanding through the stem, wilting, lodging, and plant death (Fig. 1A). Some plants generated secondary roots above the inoculation point and, in some cases, recovered from disease (Fig. 1B). Statistical analysis revealed significant differences in lesion
AUDPC means among inbreds (ANOVA, $P<0.0001$ ). All inbreds developed moderate to severe disease symptoms, except for Mp717, which only displayed symptoms related to the wounding inflicted during inoculations (Fig. 2). Mp717 was the only inbred designated as resistant to stalk rot in our experiment for PH1 and mycotoxin mutants, and all other inbreds were significantly different from Mp717 according to lesion AUDPC data (Fig. 2). Inbreds Mp317, $\mathrm{OH} 43$, and HP301 showed low susceptibility to PH1 and mycotoxin mutants and were designated as moderately resistant (Fig. 2).

All F. graminearum isolates were capable of infecting maize inbreds and causing stalk rot regardless of their ability to produce DON and ZEA. However, significant differences in AUDPC values were detected among $F$. gramineraum strains used to inoculate inbreds (ANOVA, $P<0.0001$ ). Mean AUDPC values and disease response categories revealed that $\mathrm{PH} 1$ was the most virulent isolate, followed by $\Delta$ tri5 and $\Delta$ zea $1 \Delta$ zea 2 (Fig. 3). Inbred-isolate interactions in AUDPC data were found to be significant in our experiment (ANOVA, $P<0.0001$ ). When inbred-isolate interactions were examined in AUDPC data, inbreds GEMS-0002, GEMS0030, MS71, and OH7B were not significantly different from moderately resistant inbreds to $\mathrm{PH} 1$ when inoculated with the DON mutant $\Delta$ tri5 (Fig. 2B). Inbreds B97, GEMS-0002, GEMS-0030, M162W, MS71, and P39 were not significantly different from moderately resistant inbreds to PH1 when inoculated with the ZEA mutant $\Delta$ zea1 $\Delta$ zea2 according to lesion AUDPC data (Fig. 2C). All other inbreds were significantly different from resistant and moderately resistant inbreds to $\mathrm{PH} 1$ and designated as susceptible, with P39, LH132, and NC444 being the inbreds that displayed the most severe symptoms to $\mathrm{PH} 1, \Delta$ tri5, and $\Delta$ zea $1 \Delta$ zea2, respectively, in our experiment (Fig. 2). The isolates were always recovered from the two plants sampled for isolations for inbreds that were moderately resistant and inbreds that were susceptible; however, isolates were only recovered in the resistant inbred Mp717 for time points 2 and $14 \mathrm{dpi}$ but not for time point $28 \mathrm{dpi}$.

DON accumulation at 28 dpi was measured in representative maize inbreds of resistant, moderately resistant, and susceptible phenotypes using targeted metabolomics. As expected, DON was only detected in plants inoculated with the wild-type strain PH1 and the ZEA mutant $\Delta$ zea1 $\Delta$ zea2 but not in the ones with the DON mutant $\Delta$ tri5 and mock inoculation. There were significant differences in DON concentration among inbreds at 28 dpi (ANOVA, $P<$ 0.0001 ), with higher accumulation occurring in susceptible inbreds

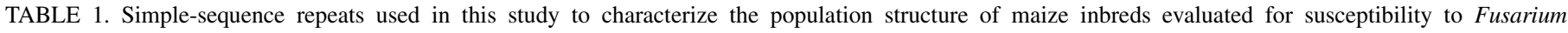
graminearum $\mathrm{PH} 1$ and mycotoxin mutants

\begin{tabular}{|c|c|c|c|c|c|c|c|c|}
\hline Name & $\mathrm{Chr}^{\mathrm{a}}$ & Forward primer & Reverse primer & $\mathrm{Tm}^{\mathrm{b}}$ & $\mathrm{Als}^{\mathrm{c}}$ & $\mathrm{GD}^{\mathrm{d}}$ & $\mathrm{PIC}^{\mathrm{e}}$ & Source $^{f}$ \\
\hline phiO02 & 1.07 & CATGCAATCAATAACGATGGCGAGT & TTAGCGTAACCCTTCTCCAGTCAGC & 58 & 2 & 0.48 & 0.36 & MaizeGDB \\
\hline umc1147 & 1.07 & GAGAAACCATCGACCCTTCCTAAC & TTCCTATGGTACAGTTCTCCCTCG & 57 & 4 & 0.53 & 0.49 & Romay \\
\hline phi011 & 1.09 & TGTTGCTCGGTCACCATACC & GCACACACACAGGACGACAGT & 57 & 3 & 0.59 & 0.51 & MaizeGDB \\
\hline umc 1725 & 1.11 & CTACGGCCAGAGTATCGGTCAC & TATGGTGGGAGAGACGACAGGTAT & 58 & 2 & 0.49 & 0.37 & Romay \\
\hline umc1605 & 1.12 & CCAGGAGAGAAATCAACAAAGCAT & GGAGAAGCACGCCTTCGTATAG & 55 & 2 & 0.50 & 0.37 & MaizeGDB \\
\hline umc1165 & $2.00-2.02$ & TATCTTCAGACCCAAACATCGTCC & GTCGATTGATTTCCCGATGTTAAA & 56 & 2 & 0.49 & 0.37 & Romay \\
\hline umc1265 & 2.02 & GCCTAGTCGCCTACCCTACCAAT & TGTGTTCTTGATTGGGTGAGACAT & 56 & 3 & 0.65 & 0.58 & Romay \\
\hline umc1185a & 2.03 & AGTAAAAGAGGCAAGGACTACGGC & GCGGCGATATATACGAGGTTGT & 58 & 2 & 0.50 & 0.37 & Romay \\
\hline umc1185b & 2.03 & AGTAAAAGAGGCAAGGACTACGGC & GCGGCGATATATACGAGGTTGT & 58 & 2 & 0.06 & 0.06 & Romay \\
\hline phi109642 & $2.03-2.04$ & СТСТCTTTCCTTCCGACTTTCC & GAGCGAGCGAGAGAGATCG & 55 & 2 & 0.49 & 0.37 & Romay \\
\hline bnlg1520 & 2.09 & TCСTCTTGCTCTCCATGTCC & ACAGCTGCGTAGCTTCTTCC & 56 & 3 & 0.62 & 0.55 & Romay \\
\hline umc1696 & 2.1 & CTAGGGTTTAACCAACGGGGAG & TAAGGAGAGGGTCGATGAACACAT & 57 & 2 & 0.45 & 0.35 & MaizeGDB \\
\hline phi453121 & $3.00-3.01$ & ACCTTGCCTGTCCTTCTTTCT & CAAGCAAGACTTTTGATCAGCC & 56 & 3 & 0.62 & 0.54 & Warburton \\
\hline phi102228 & 3.06 & ATTCCGACGCAATCAACA & TTCATCTCCTCCAGGAGCCTT & 52 & 2 & 0.40 & 0.32 & Warburton \\
\hline umc1303 & 4.05 & CTTGGTAGCTTCGTATTCGACGAG & ATCCTAGGAAAGCAGGGAGGG & 57 & 3 & 0.56 & 0.49 & Romay \\
\hline umc1109 & 4.1 & GCAACACAGGACCAAATCATCTCT & GTTCGGTCCGTAGAAGAACTCTCA & 57 & 3 & 0.34 & 0.30 & Warburton \\
\hline umc1097 & 5 & CTCGTCAACGTCAACCCAAGTAAG & CTGTTAGATGTGCGACAACAGAGC & 57 & 3 & 0.53 & 0.47 & Romay \\
\hline
\end{tabular}

a Chromosome location.

b Annealing temperature.

c Alleles.

d Genetic diversity.

e Polymorphism information content.

f Romay = Romay et al. 2012 and Warburton = Warburton et al. 2007. 
NC444, IL14H, and, B73, followed by moderately resistant inbreds HP301 and Mp317 and the resistant inbred Mp717. DON was not detected in control or $\Delta$ tri5 inoculated plants. Correlation analyses indicated significant correlation between DON content at $28 \mathrm{dpi}$ and lesion AUDPC data in inbreds Mp717, B73, IL14H, NC444, HP301, and Mp317 when infected with PH1 and $\Delta$ zea1 $\Delta$ zea2 (Pearson's correlation coefficient $=0.74, P<0.0001$ ) (Fig. 4).

All SSR tested in the maize inbreds were polymorphic, with different number of alleles, level of genetic diversity, and polymorphism information content (Table 1). In total, 43 alleles were detected among the $17 \mathrm{SSR}$, ranging from 2 to 4 alleles per locus, with an average allele diversity of 2.5 alleles per primer pair. The mean genetic diversity index of the inbreds was 0.49 , ranging from 0.06 to 0.65 . The structure analysis of SSR data detected population structure among inbreds; however, the analysis showed no direct correspondence between susceptibility to stalk rot and inferred genetic clusters (Fig. 5). A clear peak in likelihoods was observed when the number of clusters was set to seven $(K=6, \operatorname{lnP}=-456.9$; $K=7, \operatorname{lnP}=-427.3$; and $K=8, \ln \mathrm{P}=-456.2$ ). Individual ancestry coefficients were highly consistent across replicate runs. The bar plots indicated that some inbreds are highly admixed, while others belong mostly to one particular cluster (Fig. 2). Isolates belonging

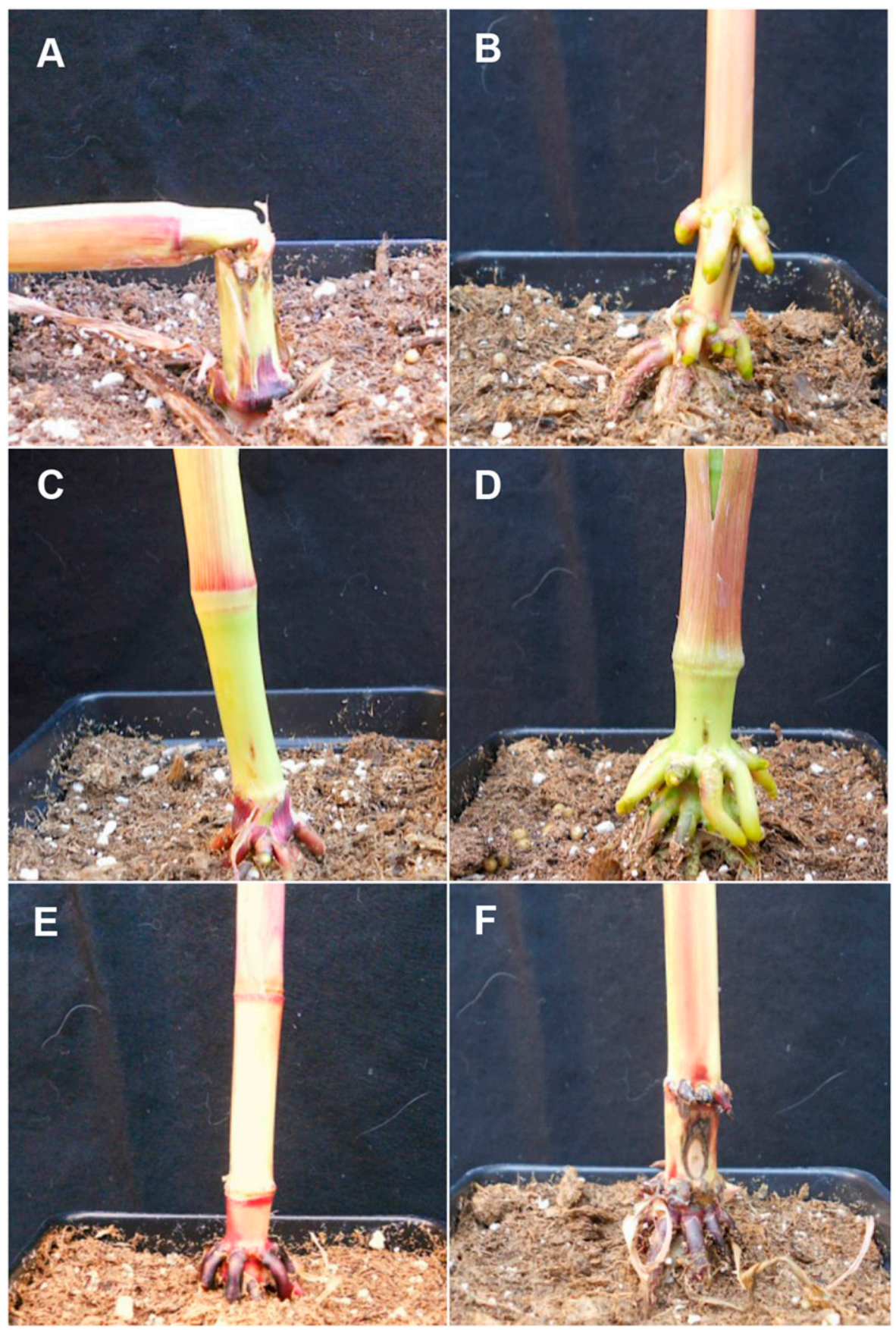

Fig. 1. Disease symptoms observed during stalk rot in maize. A, Lodging and plant death due to stalk rot in maize. B, Secondary roots generated by some maize inbreds above the inoculation point allowed them to recover from disease. C, Resistant inbred Mp717 inoculated with PH1 at 28 days postinoculation (dpi) showing only symptoms related to wounding. D, moderately resistant inbred Mp317 inoculated with PH1 at 28 dpi shows symptoms related to wounding and increased root production. E, Susceptible inbred B73 inoculated with agar as a control at 28 dpi showing symptoms related to wounding. F, Susceptible inbred B73 inoculated with PH1 at 28 dpi shows necrotic lesions at the inoculation point with sporulation, as well as necrosis of secondary roots generated above the inoculation point. 


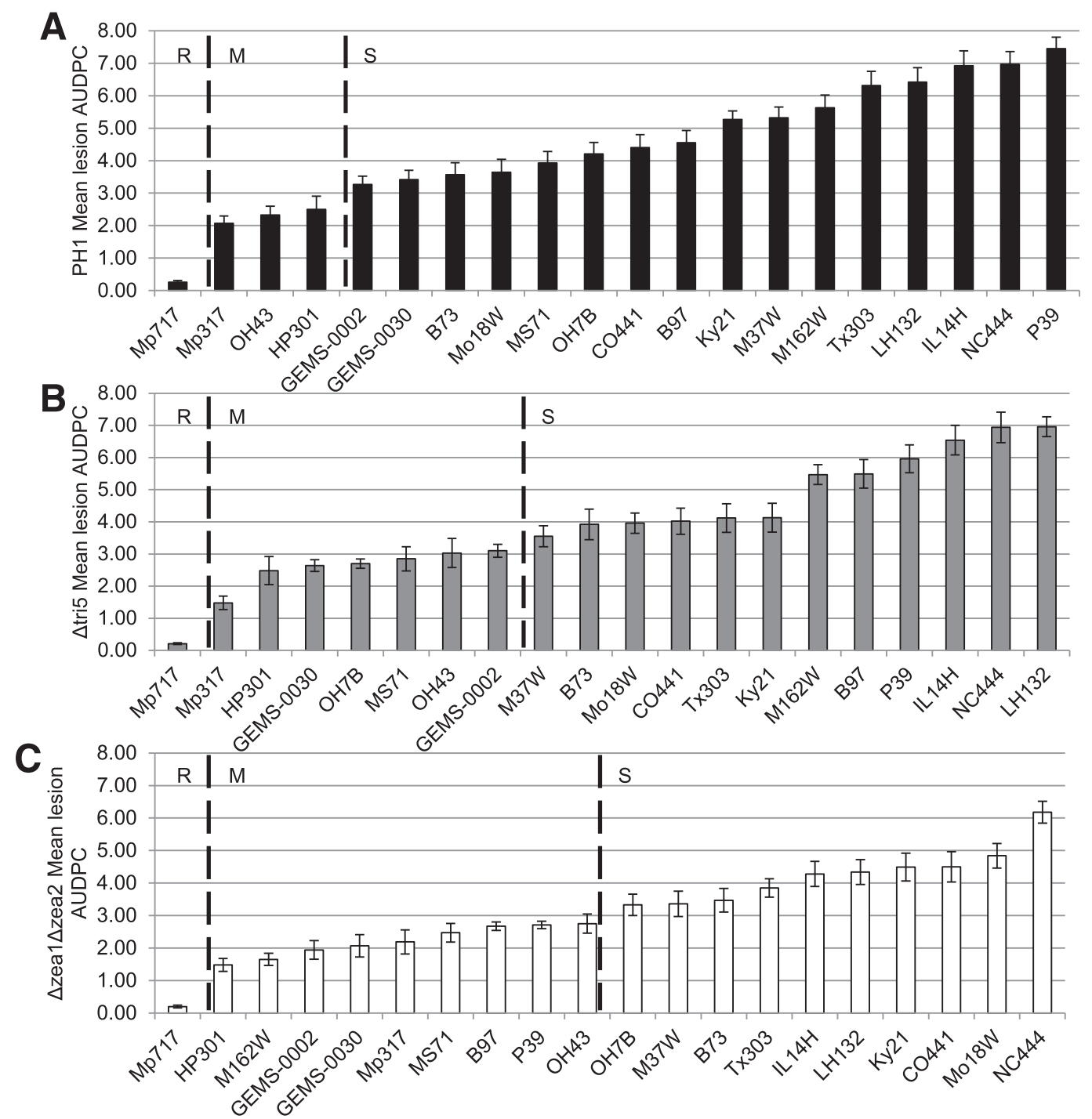

Fig. 2. Disease response of maize inbreds to Fusarium graminearum PH1 and deoxynivalenol ( $\Delta$ tri5) and zearalenone $(\Delta$ zea $1 \Delta$ zea2) mutants according to mean lesion area under the disease progress curve (AUDPC) values. A, Black columns represent AUDPC values for isolate PH1; B, gray columns for isolate $\Delta$ tri5; and $\mathbf{C}$, white columns for isolate $\Delta$ zea1 $\Delta$ zea2. Inbred lines are sorted by lowest to highest susceptibility to each isolate and susceptibility categories $(\mathrm{S}=$ susceptible, $\mathrm{M}=$ moderately resistant, and $\mathrm{R}=$ resistant) are separated by a dashed line.

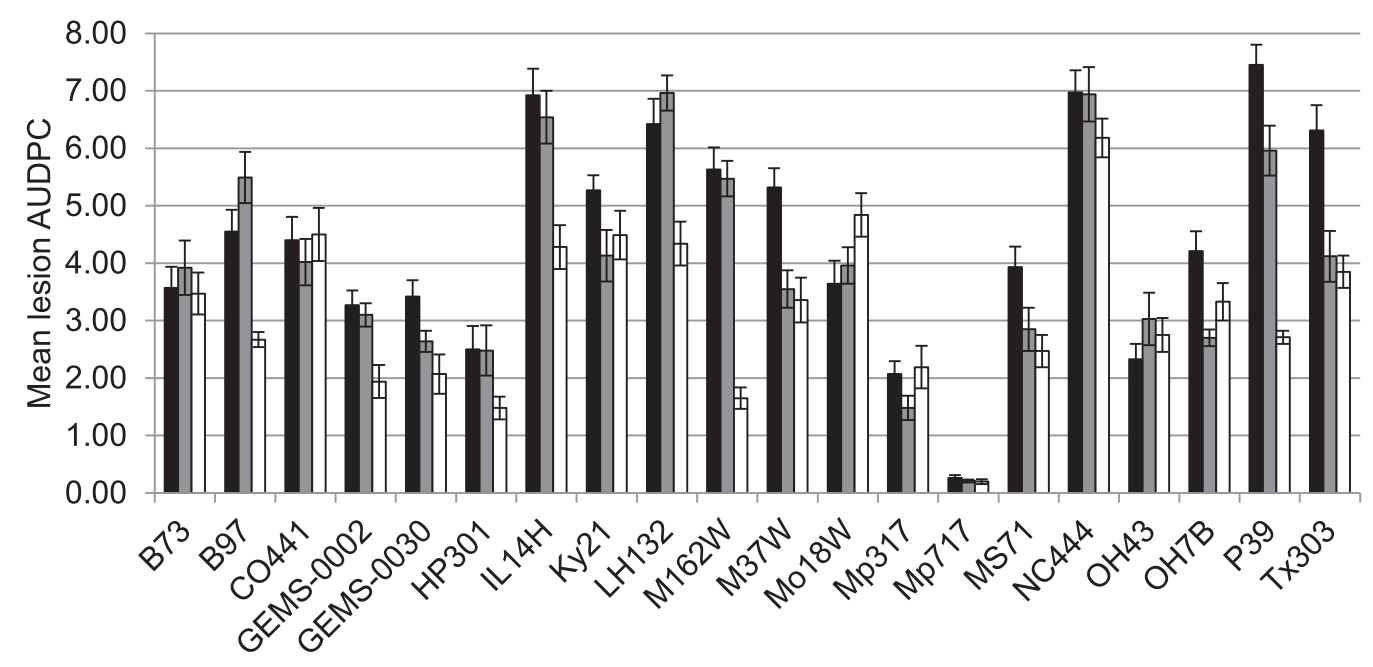

Fig. 3. Disease response of maize inbreds to Fusarium graminearum PH1 and deoxynivalenol ( $\Delta$ tri5) and zearalenone $(\Delta$ zea1 $\Delta$ zea2) mutants according to mean lesion area under the disease progress curve (AUDPC) values. Black columns represent AUDPC values for isolate PH1, gray columns for isolate $\Delta$ tri5, and white columns for isolate $\Delta$ zea $1 \Delta$ zea 2 . 
to clusters one (red), three (blue), and four (brown) presented fewer admixture than individuals from other clusters. The resistant inbred Mp717 had membership predominantly in cluster four. The moderately resistant inbred Mp317 was highly admixed while HP301 had membership predominantly in cluster two and OH43 in cluster six. Inbreds moderately resistant to mycotoxin mutants but not PH1 had predominant membership in clusters one, four, five, six, and seven or were admixed. No inbreds showing complete or moderate resistance to stalk rot were found with predominant membership in cluster three. Inbreds susceptible to stalk rot and with predominant membership in one cluster were found in genetic clusters one, two, three, four, and five. When compared with the UPGMA dendrogram, genetic clusters largely overlapped, with the grouping of dendrogram branches based on genetic distance. Maize inbreds belonging mostly to a particular genetic cluster were also grouped together in the dendrogram, whereas moderate to highly admixed individuals were found in different parts of the dendrogram.

\section{DISCUSSION}

In the present study, only one inbred was found to be resistant (Mp717), while three inbreds were moderately resistant (HP301, $\mathrm{Mp} 317$, and $\mathrm{OH} 43$ ) and the remaining 16 inbreds were susceptible to PH1. Interestingly, earlier evaluation of another group of 20 inbreds found Mp717 to be most resistant to ear rot caused by F. verticillioides and mycotoxin accumulation in that panel as well (Henry et al. 2009). Inbreds Mp317 and OH43 have also shown to have higher levels of resistance to ear rot caused by $F$. verticillioides when compared with other inbreds (King and Scott 1981; Warren 1978). Previous work by Mohammadi et al. (2011), however, found that inbred CO441 was also moderately resistant to ear rot caused by $F$. graminearum and showed stronger upregulation of defense genes and reduced DON accumulation upon inoculation compared with inbred B73, which was susceptible in their experiments. Our study, however, suggests no significant difference between inbreds CO441 and B73 in susceptibility to stalk rot caused by $F$. graminearum or in DON accumulation. This may be due to differences in tissue type and pathogen isolate responses in CO441 to ear rot versus stalk rot, or could be due to the use of different isolates for disease evaluation in both studies.

DON accumulation is significantly variable among the tested inbreds and is positively correlated with visual symptom scoring of disease severity. Previous investigations into host resistance to ear rot, also caused by F. graminearum, have found similar results. Both partial resistance (Cullen 1983; Gendloff et al. 1986) and major gene resistance (Reid et al. 1994) have been identified in maize to ear rot, and resistant inbreds displaying reduced levels of disease also have shown to have reduced levels of DON accumulation (Reid et al. 1994). A study by Atlin et al. (1983), also investigating the role of host genotype on maize ear rot and mycotoxin accumulation, found comparable results, because DON and ZEA levels both correlated with disease levels. Similarly, in wheat, a higher percentage of kernels and spikelets infected with $F$. graminearum was shown to correlate with higher DON concentrations (Bai et al. 2001). Our study identified resistance to stalk rot and DON accumulation in inbreds commonly used in breeding programs. The finding that lesion area of stalk rot in maize is correlated with mycotoxin accumulation is consistent with previous studies. Detailed studies confirming this correlation between DON and lesion area would facilitate the breeding of resistant maize germplasm if mycotoxin concentration could be used as phenotypic marker for disease level in high-throughput metabolite phenotyping.

$F$. graminearum produces several mycotoxins, including DON, NIV and its derivatives, ZEA, fusarin C, fusaric acid, and aurofusarin (Trail 2009). Trichothecenes such as DON and NIV are also potent phytotoxins that constitute virulence factors in wheat (Desjardins et al. 1996) and, to some extent, in maize during ear rot (Harris et al. 1999). Here, we demonstrate significant differences in virulence associated with ZEA and DON production, with the ability to produce both conferring the highest virulence when infecting the inbreds tested here. This result is consistent with similar reports on maize ear rot, because Harris et al. (1999) found that, although DON mutant strains were still able to cause visual disease symptoms, symptoms were less severe as pathogen virulence was reduced. Studies in wheat have shown that trichothecene mutant strains are able to initiate infection of wheat heads and wheat crowns but are less virulent because symptoms, colonization of stems, and overall disease levels are lower than that of a wild-type isolate (Desjardins et al. 1996; Mudge et al. 2006). In our study, the inbred-isolate interaction was also significant, indicating a differential response to mycotoxin mutants based upon host genotype. Four (GEMS-0030, GEMS-0002, MS71, and OH7B) and six (B97, P39, GEMS-0002, GEMS-0030, M162W, and MS71) inbreds that were susceptible to PH1 were moderately resistant to $\Delta$ tri5 and $\Delta$ zea1 $\Delta$ zea2, respectively, suggesting that DON and ZEA could be virulence factors in maize.

SSR markers were chosen to access genotypic diversity and population structure within the selected inbreds because they have shown to provide sufficient population clustering resolution in previous studies, when compared with single-nucleotide polymorphism (SNP) markers (Hamblin et al. 2007). The low allele

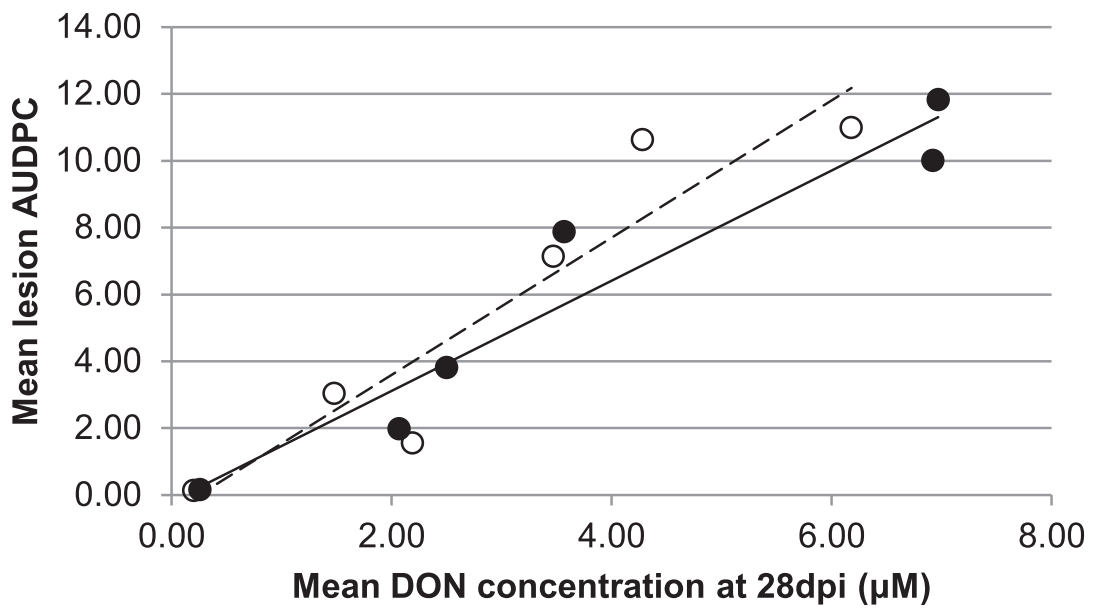

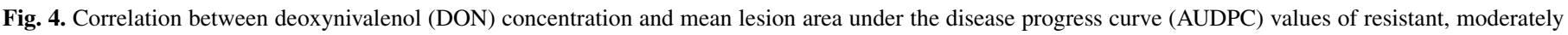

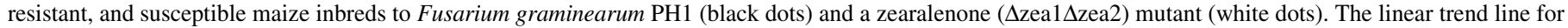
PH1 is represented by a solid line and for $\Delta$ zea1 $\Delta$ zea2 by a dashed line (Pearson's correlation coefficient $=0.74, P<0.0001)$. 


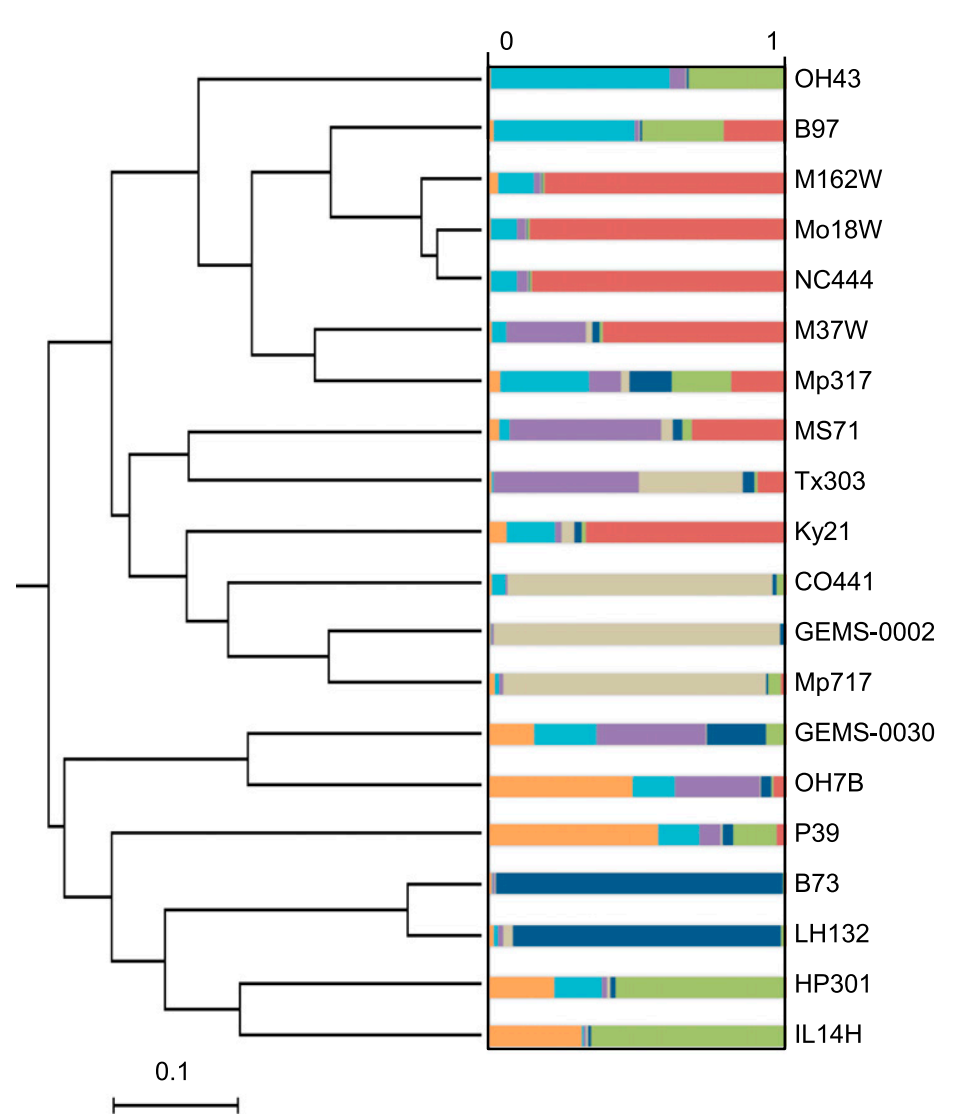

\begin{tabular}{ccc} 
PH1 & $\Delta$ tri5 & $\Delta$ zea1 $\Delta$ zea2 \\
\hline M & M & M \\
S & S & M \\
S & S & M \\
S & S & S \\
S & S & S \\
S & S & S \\
M & M & M \\
S & M & M \\
S & S & S \\
S & S & S \\
S & S & S \\
S & M & M \\
R & R & R \\
S & M & M \\
S & M & S \\
S & S & M \\
S & S & S \\
S & S & S \\
M & M & M \\
S & S & S
\end{tabular}

Fig. 5. Unweighted pair-group method with arithmetic mean (UPGMA) dendrogram and population structure of maize inbreds tested for susceptibility to stalk rot. The UPGMA dendrogram was calculated using Euclidean distance. Population structure for each inbred is represented by a bar partitioned into colored segments, each representing the individual's proportionate genetic membership in a given Kth cluster sorted numerically from right to left of the bar (i.e., cluster 1 will always be on the right of the bar and cluster 7 on the left). Cluster colors: red $=$ one, green $=$ two, blue $=$ three, brown $=$ four, purple $=$ five, aqua $=$ six, and orange $=$ seven . Susceptibility ( $\mathrm{S}=$ susceptible, $\mathrm{M}=$ moderately resistant, and $\mathrm{R}=$ resistant) to Fusarium graminearum PH1 and deoxynivalenol ( $\Delta$ tri5) and zearalenone ( $\Delta$ zea1 $\Delta$ zea2) mutants is indicated next to each inbred.

diversity in the inbreds observed here, as compared with previous studies (Liu et al. 2003), is likely the result of the limited number and the similarity of inbreds being surveyed and the inclusion of SSR containing longer repeat motifs (Vigouroux et al. 2002). The mean genetic diversity index of the 20 inbreds was 0.49 , also lower than previous reports (Liu et al. 2003), but is possibly due to similarity between the surveyed inbreds and the lack of dinucleotide repeats. Structure analysis using the data from the 17 SSR did reveal population structure among the 20 inbreds; however, there was no association between resistance to stalk rot and the genetic clusters. This is consistent with previous work on Fusarium ear rot that examined subpopulations within a set of 1,687 inbreds and found that there were no significant differences in disease severity between groups, regardless of the presence or absence of certain disease resistance-associated SNP markers (Zila et al. 2014). Numerous population studies of inbreds have been conducted previously, each based on a diverse array of markers or traits and varying groups of inbreds, and some of the same patterns were also observed in our results. Inbreds B73 and $\mathrm{OH} 43$ were found to be relatively distant in a separate study (Hansey et al. 2011), similar to what was observed in the population structure generated here. Analysis of genetic structure of 260 inbreds using microsatellites also revealed similar clustering to that which was generated in this study for the same inbreds used (Dufresne et al. 2008). Clustering of 302 inbreds based on data generated with 89 SSR markers identified comparable relationships between inbreds OH43, B73, B97, M162W, Mo18W, M37W, MS71, Tx303, Ky21, OH7B, P39, HP301, and IL14H, which were 13 of the inbreds included in the present study (Flint-Garcia et al. 2005). Comparison of genetic clusters with the UPGMA dendrogram based on genetic distances revealed much similarity in grouping, with differences only in the moderate and highly admixed inbreds. SSR used in this study were selected from the MaizeGDB website and previous publications (Lawrence et al. 2004; Romay et al. 2012; Warburton et al. 2007). Lack of association between resistance to stalk rot and genetic clustering could be due to a low number of loci surveyed or a polygenic trait. Recent development of new genomic information in maize that identified 681,257 SNP markers using genotyping by sequencing of 2,815 inbreds (Romay et al. 2013) provides a powerful resource for association mapping and identification of loci associated with stalk rot resistance.

\section{ACKNOWLEDGMENTS}

We thank all members of the Quesada lab for their valuable help, especially S. Withers for assistance with microsatellite genotyping; and E. Crisovan, B. Vaillancourt, M., M. Hardin, and A. Boone for technical, field, and greenhouse assistance. This project was supported by the Agriculture and Food Research Initiative Competitive Grant number 201267012-19810, United States Department of Agriculture-Agricultural Research Service project number NC02418, and Michigan State University Agbioresearch.

\section{LITERATURE CITED}

Atlin, G. N., Enerson, P. M., McGirr, L. G., and Hunter, R. B. 1983. Gibberella ear rot development and zearalenone and vomitoxin production as affected by maize genotype and Gibberella zeae strain. Can. J. Plant Sci. 63:847-853.

Bai, G. H., Plattner, R., Desjardins, A., and Kolb, F. 2001. Resistance to Fusarium head blight and deoxynivalenol accumulation in wheat. Plant Breed. 120:1-6.

Baldwin, T. K., Gaffoor, I., Antoniw, J., Andries, C., Guenther, J., Urban, M., Hallen-Adams, H., Pitkin, J., Hammond-Kosack, K., and Trail, F. 2010. 
A partial chromosomal deletion caused by random plasmid integration resulted in a reduced virulence phenotype in Fusarium graminearum. Mol. Plant-Microbe Interact. 23:1083-1096.

Cullen, D., Caldwell, R. W., and Smalley, E. B. 1983. Susceptibility of maize to Gibberella zeae ear rot: Relationship to host genotype, pathogen virulence, and zearalenone contamination. Plant Dis. 67:89-91.

Cuomo, C. A., Guldener, U., Xu, J. R., Trail, F., Turgeon, B. G., Di Pietro, A., Walton, J. D., Ma, L. J., Baker, S. E., Rep, M., Adam, G., Antoniw, J., Baldwin, T., Calvo, S., Chang, Y. L., DeCaprio, D., Gale, L. R., Gnerre, S., Goswami, R. S., Hammond-Kosack, K., Harris, L. J., Hilburn, K., Kennell, J. C., Kroken, S., Magnuson, J. K., Mannhaupt, G., Mauceli, E., Mewes, H. W., Mitterbauer, R., Muehlbauer, G., Munsterkotter, M., Nelson, D., O'Donnell, K., Ouellet, T., Qi, W., Quesneville, H., Roncero, M. I. G., Seong, K. Y., Tetko, I. V., Urban, M., Waalwijk, C., Ward, T. J., Yao, J., Birren, B. W., and Kistler, H. C. 2007. The Fusarium graminearum genome reveals a link between localized polymorphism and pathogen specialization. Science 317:1400-1402.

Desjardins, A. E., Proctor, R. H., Guihua, B., McCormick, S. P., Shaner, G., Buechley, G., and Hohn, T. M. 1996. Reduced virulence of trichothecenenonproducing mutants of Gibberella zeae in wheat field tests. Mol. PlantMicrobe Interact. 9:775-781.

Dorn, B., Forrer, H. R., Jenny, E., Wettstein, F. E., Bucheli, T. D., and Vogelgsang, S. 2011. Fusarium species complex and mycotoxins in grain maize from maize hybrid trials and from growers fields. J. Appl. Microbiol. 111:693-706.

Dufresne, M., van der Lee, T., M'Barek, S. B., Xu, X., Zhang, X., Liu, T., Waalwijk, C., Zhang, W., Kema, G. H. J., and Daboussi, M.-J. 2008. Transposon-tagging identifies novel pathogenicity genes in Fusarium graminearum. Fungal Genet. Biol. 45:1552-1561.

Eckard, S., Wettstein, F. E., Forrer, H. R., and Vogelgsang, S. 2011. Incidence of Fusarium species and mycotoxins in silage maize. Toxins (Basel) 3:949-967.

Flint-Garcia, S. A., Thuillet, A.-C., Yu, J., Pressoir, G., Romero, S. M., Mitchell, S. E., Doebley, J., Kresovich, S., Goodman, M. M., and Buckler, E. S. 2005. Maize association population: A high-resolution platform for quantitative trait locus dissection. Plant J. 44:1054-1064.

Gaffoor, I., Brown, D. W., Plattner, R., Proctor, R. H., Qi, W., and Trail, F. 2005. Functional analysis of the polyketide synthase genes in the filamentous fungus Gibberella zeae (anamorph Fusarium graminearum). Eukaryot. Cell 4:1926-1933.

Gaffoor, I., and Trail, F. 2006. Characterization of two polyketide synthase genes involved in zearalenone biosynthesis in Gibberella zeae. Appl. Environ. Microbiol. 72:1793-1799.

Gendloff, E. H., Rossman, E. C., Casale, W. L., Isleib, T. G., and Hart, L. P. 1986. Components of resistance to Fusarium ear rot in field corn. Phytopathology 76:684-688.

Goswami, R. S., and Kistler, H. C. 2004. Heading for disaster: Fusarium graminearum on cereal crops. Mol. Plant Pathol. 5:515-525.

Hamblin, M. T., Warburton, M. L., and Buckler, E. S. 2007. Empirical comparison of simple sequence repeats and single nucleotide polymorphisms in assessment of maize diversity and relatedness. PLoS One 2:e1367.

Hansey, C. N., Johnson, J. M., Sekhon, R. S., Kaeppler, S. M., and Leon, N. d. 2011. Genetic diversity of a maize association population with restricted phenology. Crop Sci. 51:704-715.

Harris, L. J., Desjardins, A. E., Plattner, R. D., Nicholson, P., Butler, G., Young, J. C., Weston, G., Proctor, R. H., and Hohn, T. M. 1999. Possible role of trichothecene mycotoxins in virulence of Fusarium graminearum on maize. Plant Dis. 83:954-960.

Hart, L. P., Braselton, W. E., and Stebbins, T. C. 1982. Production of zearalenone and deoxynivalenol in commercial sweet corn. Plant Dis. 66: 1133-1135.

Henry, W. B., Williams, W. P., Windham, G. L., and Hawkins, L. K. 2009. Evaluation of maize inbred lines for resistance to Aspergillus and Fusarium ear rot and mycotoxin accumulation. Agron. J. 101:1219-1226.

Kazan, K., Gardiner, D. M., and Manners, J. M. 2012. On the trail of a cereal killer: Recent advances in Fusarium graminearum pathogenomics and host resistance. Mol. Plant Pathol. 13:399-413.

King, S. B., and Scott, G. E. 1981. Genotypic differences in maize to kernel infection by Fusarium moniliforme. Phytopathology 71:1245-1247.

Lawrence, C. J., Dong, Q., Polacco, M. L., Seigfried, T. E., and Brendel, V. 2004. MaizeGDB, the community database for maize genetics and genomics. Nucleic Acids Res. 32:D393-D397.

Liu, K., Goodman, M., Muse, S., Smith, J. S., Buckler, E., and Doebley, J. 2003. Genetic structure and diversity among maize inbred lines as inferred from DNA microsatellites. Genetics 165:2117-2128.
Liu, K., and Muse, S. V. 2005. PowerMarker: An integrated analysis environment for genetic marker analysis. Bioinformatics 21:2128-2129.

Lysøe, E., Seong, K. Y., and Kistler, H. C. 2011. The transcriptome of Fusarium graminearum during the infection of wheat. Mol. Plant-Microbe Interact. 24:995-1000.

McMullen, M. D., Kresovich, S., Sanchez-Villeda, H., Bradbury, P., Li, H., Sun, Q., Flint-Garcia, S., Thornsberry, J., Acharya, C., Bottoms, C., Brown, P., Browne, C., Eller, M., Guill, K., Harjes, C., Kroon, D., Lepak, N., Mitchell, S. E., Peterson, B., Pressoir, G., Romero, S., Oropeza-Rosas, M., Salvo, S., Yates, H., Hanson, M., Jones, E., Smith, S., Glaubitz, J. C., Goodman, M., Ware, D., Holland, J. B., and Buckler, E. S. 2009. Genetic properties of the maize nested association mapping population. Science 325:737-740.

Mirocha, C. J., and Christensen, C. M. 1974. Fungus metabolites toxic to animals. Annu. Rev. Phytopathol. 12:303-330.

Mohammadi, M., Anoop, V., Gleddie, S., and Harris, L. J. 2011. Proteomic profiling of two maize inbreds during early Gibberella ear rot infection. Proteomics 11:3675-3684.

Mudge, A. M., Dill-Macky, R., Dong, Y., Gardiner, D. M., White, R. G., and Manners, J. M. 2006. A role for the mycotoxin deoxynivalenol in stem colonisation during crown rot disease of wheat caused by Fusarium graminearum and Fusarium pseudograminearum. Physiol. Mol. Plant Pathol. 69:73-85.

Munkvold, G. P. 2003. Cultural and genetic approaches to managing mycotoxins in maize. Annu. Rev. Phytopathol. 41:99-116.

Proctor, R. H., Hohn, T. M., and McCormick, S. P. 1995. Reduced virulence of Gibberella zeae caused by disruption of a trichothecene toxin biosynthetic gene. Mol. Plant-Microbe Interact. 8:593-601.

R Core Team. 2013. R: A Language and Environment for Statistical Computing. R Foundation for Statistical Computing, Vienna.

Reid, L., Mather, D., Bolton, A., and Hamilton, R. 1994. Evidence for a gene for silk resistance to Fusarium graminearum Schw. ear rot of maize. J. Hered. 85:118-121.

Romay, M. C., Butrón, A., Ordás, A., Revilla, P., and Ordás, B. 2012. Effect of recurrent selection on the genetic structure of two broad-based Spanish maize populations. Crop Sci. 52:1493-1502.

Romay, M. C., Millard, M. J., Glaubitz, J. C., Peiffer, J. A., Swarts, K. L., Casstevens, T. M., Elshire, R. J., Acharya, C. B., Mitchell, S. E., and Flint-Garcia, S. A. 2013. Comprehensive genotyping of the USA national maize inbred seed bank. Genome Biol. 14:R55.

Schuelke, M. 2000. An economic method for the fluorescent labeling of PCR fragments. Nat. Biotechnol. 18:233-234.

Seong, K., Hou, Z., Tracy, M., Kistler, H. C., and Xu, J. R. 2005. Random insertional mutagenesis identifies genes associated with virulence in the wheat scab fungus Fusarium graminearum. Phytopathology 95: 744-750.

Sutton, J. C. 1982. Epidemiology of wheat head blight and maize ear rot caused by Fusarium graminearum. Can. J. Plant Pathol. 4:195-209.

Tamura, K., Stecher, G., Peterson, D., Filipski, A., and Kumar, S. 2013. MEGA6: Molecular evolutionary genetics analysis version 6.0. Mol. Biol. Evol. 30:2725-2729.

Trail, F. 2009. For blighted waves of grain: Fusarium graminearum in the postgenomics era. Plant Physiol. 149:103-110.

Trail, F., and Common, R. 2000. Perithecial development by Gibberella zeae: A light microscopy study. Mycologia 92:130-138.

Vigouroux, Y., Jaqueth, J. S., Matsuoka, Y., Smith, O. S., Beavis, W. D., Smith, J. S. C., and Doebley, J. 2002. Rate and pattern of mutation at microsatellite loci in maize. Mol. Biol. Evol. 19:1251-1260.

Warburton, M. L., Reif, J. C., Frisch, M., Bohn, M., Bedoya, C., Xia, X. C., Crossa, J., Franco, J., Hoisington, D., Pixley, K., Taba, S., and Melchinger, A. E. 2007. Genetic diversity in CIMMYT nontemperate maize germplasm: Landraces, open pollinated varieties, and inbred lines. Crop Sci. 48:617624.

Warren, H. 1978. Comparison of normal and high-lysine maize inbreds for resistance to kernel rot caused by Fusarium moniliforme. Phytopathology 68:1331-1335.

Ye, J., Guo, Y., Zhang, D., Zhang, N., Wang, C., and Xu, M. 2013. Cytological and molecular characterization of quantitative trait locus qRfg1, which confers resistance to Gibberella stalk rot in maize. Mol. Plant-Microbe Interact. 26:1417-1428.

Zila, C. T., Ogut, F., Romay, M. C., Gardner, C. A., Buckler, E. S., and Holland, J. B. 2014. Genome-wide association study of Fusarium ear rot disease in the USA maize inbred line collection. BMC Plant Biol. $14: 372$ 